\title{
Integrated Reconfigurable High-Voltage Transmitting Circuit for CMUTs
}

\author{
Llimos Muntal, Pere; Larsen, Dennis Øland; Jørgensen, Ivan Harald Holger; Bruun, Erik
}

Published in:

Proceedings of the 32th IEEE Norchip Conference 2014

Link to article, DOI:

10.1109/norchip.2014.7004727

Publication date:

2014

Link back to DTU Orbit

Citation $(A P A)$ :

Llimos Muntal, P., Larsen, D. Ø., Jørgensen, I. H. H., \& Bruun, E. (2014). Integrated Reconfigurable HighVoltage Transmitting Circuit for CMUTs. In Proceedings of the 32th IEEE Norchip Conference 2014 IEEE. https://doi.org/10.1109/norchip.2014.7004727

\section{General rights}

Copyright and moral rights for the publications made accessible in the public portal are retained by the authors and/or other copyright owners and it is a condition of accessing publications that users recognise and abide by the legal requirements associated with these rights.

- Users may download and print one copy of any publication from the public portal for the purpose of private study or research.

- You may not further distribute the material or use it for any profit-making activity or commercial gain

- You may freely distribute the URL identifying the publication in the public portal 


\title{
Integrated Reconfigurable High-Voltage Transmitting Circuit for CMUTs
}

\author{
Pere Llimós Muntal, Dennis Øland Larsen, Ivan H.H. Jørgensen and Erik Bruun \\ Department of Electrical Engineering \\ Technical University of Denmark, Kgs. Lyngby, Denmark \\ plmu@elektro.dtu.dk, deno@elektro.dtu.dk, ihhj@elektro.dtu.dk, eb@elektro.dtu.dk
}

\begin{abstract}
In this paper a full high-voltage transmitting circuit aimed for capacitive micromachined ultrasonic transducers (CMUTs) used in ultrasound medical applications is designed and implemented in a $0.35 \mu \mathrm{m}$ high-voltage CMOS process. The CMUT is single-ended driven. The design is taped-out and measurements are performed on the integrated circuit. The transmitting circuit is reconfigurable externally making it able to drive a wide variety of CMUTs. The transmitting circuit can generate several pulse shapes, pulse voltages up to $100 \mathrm{~V}$, maximum pulse range of $50 \mathrm{~V}$ and frequencies up to $5 \mathrm{MHz}$. The area occupied by the design is $0.938 \mathrm{~mm}^{2}$ and the maximum power consumption is $187.7 \mathrm{~mW}$.
\end{abstract}

\section{INTRODUCTION}

Ultrasound imaging systems are widely used in medical applications since it is a cost efficient, ionizing radiation free and noninvasive diagnostic technique that allows real time imaging. The complexity of ultrasound systems has been increasing throughout the years and a tendency of high integration has enabled portable ultrasound systems with comparable performance to the traditional static ultrasound systems. In Fig. 1 the typical block structure of an ultrasound system can be seen. The transmitting circuit (Tx) drives the transducer in order to generate the ultrasound, which will be reflected off of the scanned body and travel back to the transducer inducing a current that is amplified by the receiving circuit $(\mathrm{Rx})$. The amplified signal will be sent to a signal processing unit to obtain the real time imaging.

Piezoelectric transducers have been typically used in ultrasound systems, but in the last two decades extensive research has proved that capacitive micromachined ultrasonic transducers (CMUTs) are a very suitable alternative. The performance and the fabrication process are the main advantages of the CMUTs compared to the conventional piezoelectric transducers. CMUTs have a wider bandwidth, which translates into

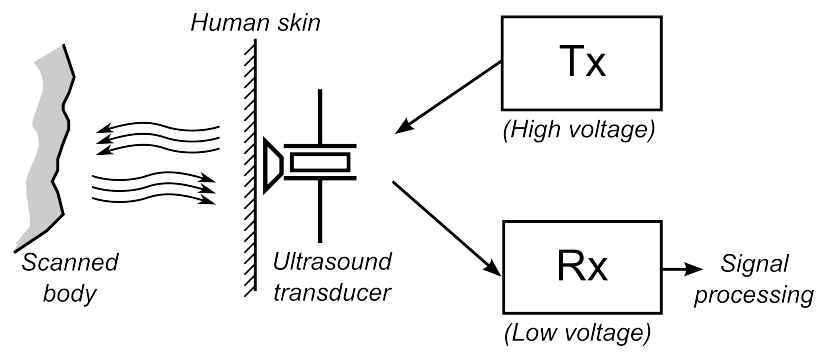

Fig. 1. Typical block structure of an ultrasound system. better temporal and axial resolution, and better thermic and transduction efficiency [1]. Moreover, they also benefit from the standard silicon integrated circuit fabrication technology advantages such as low cost and high flexibility, which allows easier fabrication of large complex transducer arrays. The last advantage of CMUTs is its high integration compatibility with electronic circuits, since CMUTs can be directly bonded with the integrated circuit die or even built on the top of a finished electronic wafer [2].

In order to operate, CMUTs require a high bias voltage between its plates in the order of $100 \mathrm{~V}$ for both receiving and transmitting. However, in transmitting mode, a high voltage pulse on the top of this bias voltage is applied to create the ultrasound. The transmitting circuitry is required to operate in high voltage, generating the bias voltage and the pulses. The bias voltage and the pulse characteristics, such as amplitude and frequency, depend on the specific CMUT to drive, therefore each transmitting circuit has to be designed and adjusted to match the requirements of the transducer.

An ultrasound scanner contains arrays of up to thousands of CMUTs that each needs a transmitting circuit. Consequently, the power consumption and area of a single transmitting circuit is key in order to make them scalable into a portable hand held scanner. Integrating the transmitting circuit in an ASIC reduces the area and the power consumption of the Tx since it is specifically designed for its application. However, the transmitting circuit requires voltages around hundred volts which can not be handled by standard CMOS processes. The $\mathrm{Tx}$ needs to be designed in a high voltage process which are significantly different from standard ones. These processes have more strict design rules since they require guard-rings and more spacing to avoid high voltage breakdowns and also use high voltage devices which are more complex than standard MOSFET devices.

This paper deals with the design and implementation of a full integrated reconfigurable transmitting circuit. It is decided to design the transmitting circuit to be reconfigurable in order to drive CMUTs with different characteristics. The bias voltage, pulse amplitude, frequency and shape are going to be adjustable externally. However, this driving flexibility has an area and power consumption cost. Nonetheless, the primary focus of this paper is to design a Tx that can generate a wide variety of driving pulses, so the area and power consumption cost is assumed and acknowledged as not being the main strength of the design. In the future, for the implementation of the Tx in the portable scanner, the area and power consumption 


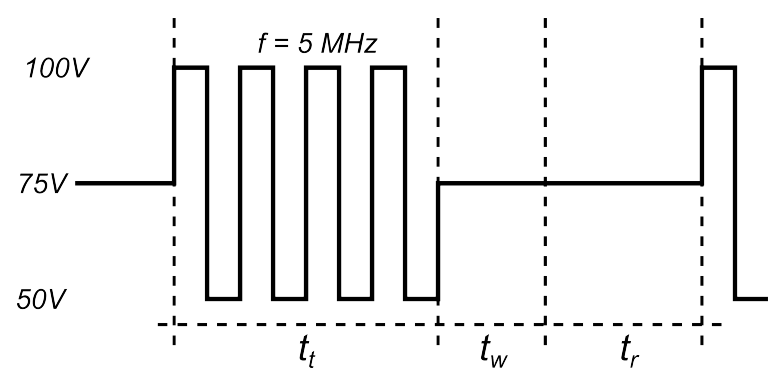

Fig. 2. Full operating cycle of the voltage between terminals of the CMUT.

can be reduced by designing the circuit for a specific CMUT.

The paper is structured as follows: In section II the specifications of the Tx circuit are defined and the topologies and blocks used to implement it are shown in section III. The layout of the integrated circuit and the measurement results can be seen in section IV and the conclusions and future work can be found in section V.

\section{TRANSMITTING CIRCUIT SPECIFICATIONS}

As it was stated before, the CMUT characteristics dictate the specifications for the transmitting circuit. In order to set the specifications for a reconfigurable transmitting circuit the most demanding transducer to be driven needs to be defined. The Tx is designed for this transducer while ensuring that it is easily reconfigurable and can function within a range of lower requirements. A CMUT is characterized by its own resonant frequency, bias voltage and pulse amplitude, which correspond to the frequency of the pulses and voltage levels that the Tx circuit needs to generate. The most demanding transducer that this Tx circuit was targeted to drive has a resonant frequency of $5 \mathrm{MHz}$, bias voltage of $75 \mathrm{~V}$ and pulse amplitude of $50 \mathrm{~V}$, which translates into voltage level generation of $50 \mathrm{~V}, 75 \mathrm{~V}$ and $100 \mathrm{~V}$.

The operating cycle of a transducer consists of a transmitting time, a waiting time and a receiving time. During transmitting time the Tx circuit is required to send to the CMUT pulses on the top of the bias voltage. In the waiting and receiving time the Tx circuit only biases the CMUT. Using the previous specifications defined by the most restrictive transducer, the voltage between the terminals of the CMUT for a full operating cycle can be seen in Fig. 2. When transmitting $\left(t_{t}\right)$, the voltage toggles between $50 \mathrm{~V}$ and $100 \mathrm{~V}$ with a frequency of $5 \mathrm{MHz}$ and during waiting $\left(t_{w}\right)$ and receiving time $\left(t_{r}\right)$ the CMUT is biased at $75 \mathrm{~V}$. This is the most demanding output signal that the transmitting circuit needs to generate. Due to these high voltage requirements the process used for the implementation of this transmitting circuit is a $0.35 \mu \mathrm{m}$ high-voltage CMOS process.

\section{DESIGN AND IMPLEMENTATION OF THE TX}

Block structure of the Tx circuit designed is shown in Fig. 3 . The inputs of the system are low voltage signals defining the frequency operation, the waiting time, the transmitting and receiving time, which are transformed by the logic block into the internal signals that the Tx circuit requires. Using the level shifter block, the low voltage signals are converted into the high voltage signals that the output stage needs in order to

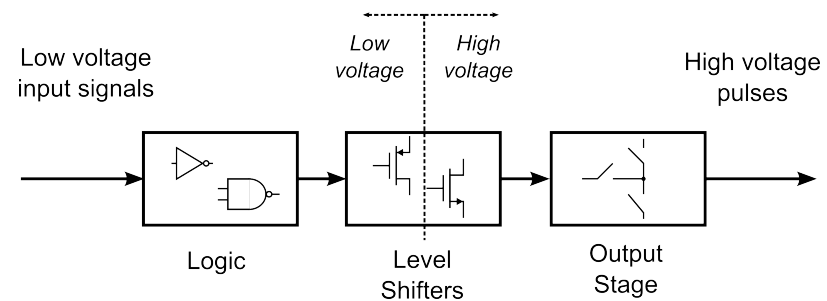

Fig. 3. Block structure of the Tx circuit.

generate the high voltage output signal described in section II. For the design of each block, high-voltage devices with different capabilities are used. In Fig. 4 the specifications and symbols for each device are shown. Note that all the MOSFET devices have the body terminal connected to the source. In the next subsections each block implementation and operation are described.

\section{A. Output stage}

The output stage drives one of the terminals of the CMUT while the second terminal is voltage biased. Since CMUTs are affected by differential voltage between their plates the main discussion is whether the biased terminal of the transducer should be high-voltage biased or grounded. High-voltage biasing one of the terminals of the CMUT has the advantage of lowering the voltage levels of the CMUT terminal connected to the output stage, hence the circuit requirements are lower and the area and power consumption are reduced. However, ultrasound scanners are used directly onto patients therefore having high voltages towards them is dangerous. For safety reasons, despite the higher voltages necessity in the output stage, in this design the terminal of the CMUT towards the patient was grounded and the output stage operates in the other terminal.

The schematic of the output stage used can be seen in Fig. 5. The MOSFETs $M_{1}-M_{2}, M_{3}-M_{4}$ and $M_{5}-M_{6}$ function as switches connecting the CMUT to $V_{C M U T, H I}=100 \mathrm{~V}$, $V_{C M U T, L O}=50 \mathrm{~V}$ and $V_{C M U T, M I D}=75 \mathrm{~V}$ respectively. The only difference between pulling the output node with $M_{1}$ and $M_{3}$ or with $M_{2}$ and $M_{4}$ is the driving speed. The resistors $R_{2}$ and $R_{4}$ are connected in series with $M_{2}$ and $M_{4}$ obtaining a slower response of the output node. This is a versatility feature that allows two different driving speeds both for the rising and falling edges of the pulses. The resistor $R_{6}$ connected in series with $M_{6}$ is added in order to increase the impedance of that node for receiving purposes. Three different voltage levels are connected to the same output node hence two switches connected to $V_{C M U T, M I D}\left(M_{5}\right.$ and $\left.M_{6}\right)$ are required in order to pull down from $V_{C M U T, H I}$ or pull up from $V_{C M U T, L O}$. To

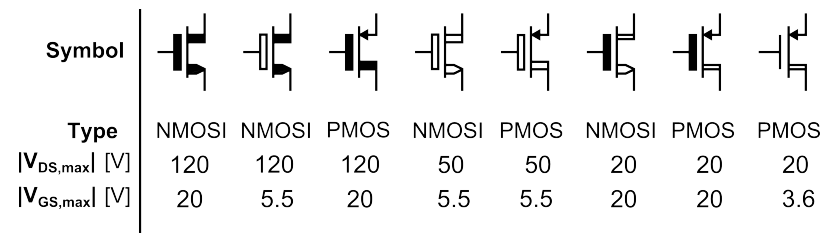

Fig. 4. High-voltage MOSFETs specifications and symbols. Note that NMOSI are isolated NMOS. 


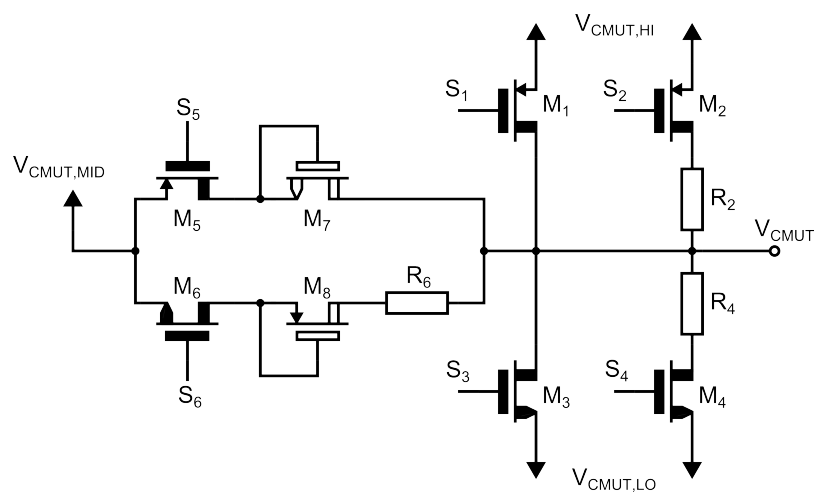

Fig. 5. Schematic of the output stage.

avoid short circuiting $V_{C M U T, H I}$ and $V_{C M U T, M I D}$ through the body diode of $M_{5}$ when the output voltage is $V_{C M U T, H I}$, the transistor $M_{7}$ acting as a diode is needed. Similarly, $M_{8}$ prevents shorting $V_{C M U T, L O}$ and $V_{C M U T, M I D}$ through the body diode of $M_{6}$ when the output voltage is $V_{C M U T, L O}$. Due to the high voltage swing between voltage levels, the output stage MOSFETs need to have strong driving capabilities which translates into high width to length ratio.

The high voltage signals $S_{1}, S_{2}, S_{3}$ and $S_{4}$ control which of the output stage MOSFETs is on at every part of the transmitting-receiving cycle. It is important to notice that only one of the MOSFETs should be on at a time, otherwise two voltage supplies are going to be shorted. During transmission $M_{1}-M_{2}$ and $M_{3}-M_{4}$ are inversely toggled on and off, in the waiting time only $M_{3}$ is turned on and in receiving time only $M_{4}$ is turned on.

\section{B. Level shifters}

The control signals of the output stage MOSFETs need to be high voltage, therefore level shifters are required. The level shifter topology used is a pulse-triggered topology and it can be seen in Fig. 6. It consist of a latch formed by $M_{17}-M_{20}$ and two branches to control the latch formed by $M_{9}, M_{11}$, $M_{13}, M_{15}$ and $M_{10}, M_{12}, M_{14}, M_{16}$. By sending a small impulse to $S_{\text {reset }}$, the first branch pulls $V_{O S}$ to $V_{L O}$ and it is maintained there by the latch. Similarly, by sending a small impulse to $S_{s e t}$, the second branch pulls $V_{O S}$ to $V_{H I}$ and it is maintained there by the latch. The main advantage of this pulse-triggered topology is the fact that it only spends current during the transitions, when the latch needs to change state. Once the latch level is established, the consumption of the level shifter is zero. The downside of this topology is that the latch needs to be very carefully designed in order to correctly define its starting state. This state should match the voltage that turns off the output stage MOSFET connected to that level shifter. If the starting state is the incorrect one, several output stage MOSFETs might be turned on during the start up which would short circuit two voltage sources.

The full transmitting circuit requires one level shifter for each output stage MOSFET, hence a total of six level shifters are used in the design. Each of them operates in different $V_{L O}$ and $V_{H I}$ according to the MOSFET that they are driving. In order to minimize the number of voltage supplies needed for the transmitting circuit the gate-source voltage range of each

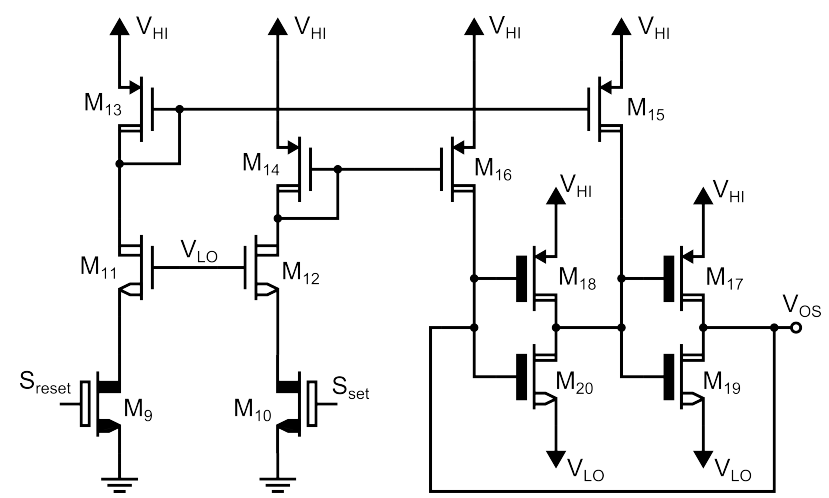

Fig. 6. Schematic of the level shifter.

MOSFET is set to $12.5 \mathrm{~V}$. The output voltages of each of the six level shifters are shown in table I.

\section{Low voltage logic}

The inputs of the Tx circuit carry the information of the pulsing frequency and the waiting, receiving and transmitting time. The functionality of the low voltage logic block is to translate these inputs into the low voltage signals for the level shifters to correctly drive the output stage. Firstly, the low voltage equivalent of the output stage control signals are generated from the inputs of the Tx. Secondly, these low voltage control signals are synchronized using flip-flops, which run at double frequency of pulses, which also needs to be supplied as an input of the circuit. These flip-flops make sure that even if some small delay is previously added to the input signals due to external routing, the signals used internally in the transmitting circuit are still synchronized. Finally the low voltage control signals are fed into a pulser circuit that generates the two corresponding set and reset impulse signals for the pulse-triggered level shifters previously described.

\section{Measurement Results AND DisCUSSION}

The transmitting circuit was taped-out in a $0.35 \mu \mathrm{m}$ highvoltage process and a picture of the integrated circuit taken with a microscope is shown in Fig. 7. Area $a$ ) contains the transmitting circuit described in this paper and area $b$ ) contains two copies of the level shifters used in the design for testing and research purposes. Inside the transmitting circuit, the output stage is contained in area $c$ ), the level shifters are situated in area $d$ ) and the logic block in area $e$ ). The total area of the transmitting circuit is $0.938 \mathrm{~mm}^{2}$.

After the tapeout, a PCB was designed in order to test the functionality of the integrated circuit. The transmitting circuit was tested with the most strict frequency and voltage

TABLE I. LEVEL SHIFTERS VOLTAGES $V_{H I}$ AND $V_{L O}$

\begin{tabular}{|c|c|c|c|}
\cline { 2 - 4 } \multicolumn{1}{c|}{} & MOSFET driving & $\mathbf{V}_{\text {HI }}[\mathbf{V}]$ & $\mathbf{V}_{\text {LO }}[\mathbf{V}]$ \\
\hline Level shifer 1 & $M_{1}$ & 100 & 87.5 \\
\hline Level shifer 2 & $M_{2}$ & 100 & 87.5 \\
\hline Level shifer 3 & $M_{3}$ & 62.5 & 50 \\
\hline Level shifer 4 & $M_{4}$ & 62.5 & 50 \\
\hline Level shifer 5 & $M_{5}$ & 75 & 62.5 \\
\hline Level shifer 6 & $M_{6}$ & 87.5 & 75 \\
\hline
\end{tabular}




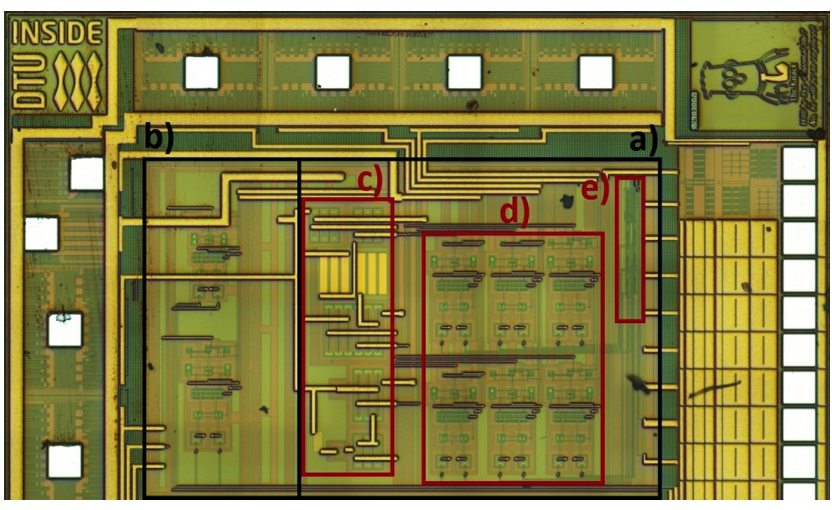

Fig. 7. Picture of the taped-out transmitting circuit. a) Tx circuit. b) Level shifters test. c) Output stage. d) Level shifters. e) Logic block.

requirements defined in section II. The transmitting, waiting and receiving times were set to $2 \mu \mathrm{s}, 0.2 \mu \mathrm{s}$ and $1.8 \mu \mathrm{s}$. The output voltage of the Tx measured on an oscilloscope is shown in Fig. 8 where the fast MOSFETs $M_{1}-M_{3}$ are used in Fig.8 a) and the slow MOSFETs $M_{2}-M_{4}$ are used in Fig. 8 b). The high-voltage transmitting circuit functions as expected, and can achieve the driving speed flexibility desired. However, in low speed, the driving strength is not enough to reach the top and bottom voltage rails. This is caused by $R_{2}$ and $R_{4}$ which were intendedly oversized in order to clearly see the slowing effect. In case that this was a critical issue for a certain transducer, $R_{2}$ and $R_{4}$ should be reduced increasing the speed and allowing the output of the Tx reach full voltage range. In order to have an idea of the power consumption of the circuit, the currents drawn from each voltage source are measured while driving a capacitive load of approximately $15 \mathrm{pF}$. The power consumption of the transmitting circuit operating at maximum requirements was $187.7 \mathrm{~mW}$.

The circuit is easily reconfigurable by setting externally different frequencies, number of pulses, waiting and receiving times and voltages. During operation, the Tx can be easily switched on and off without the need of restarting the whole setup, or even switch between $M_{1}-M_{2}$ and $M_{3}-M_{4}$ independently. The target of this paper of designing and implementing an integrated reconfigurable high-voltage transmitting circuit was achieved.

However, if this design should be used in an ultrasound scanner the power consumption and area should be reduced. Ultrasound scanners contain thousands of transmitting circuits therefore their power consumption and area need to be scalable. The first step would be to re-design the Tx circuit for the specific CMUT that the scanner is using and remove the reconfigurability features. Another approach that could be used is to reduce the gate-source voltage swing of the output stage MOSFETs. It would increase the number of DC voltage supplies needed for the circuit but it would allow to use smaller devices both in the level shifters and the output stage, which would decrease the area and lower the power consumption. Finally, it would be interesting to investigate if it is possible to add a protection to the ultrasound scanner that completely voltage-isolates the patient from the transducer and fulfills with the medical equipment standards. This isolation would allow to high-voltage bias the terminal of the CMUT facing the patient.

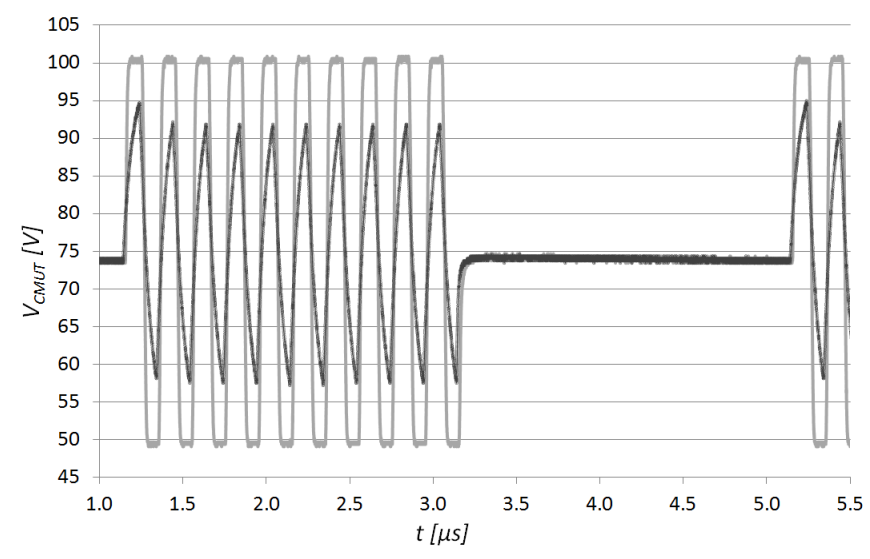

Fig. 8. Output voltage measured on the integrated circuit. a) Fast transitions in light grey. b) Slow transitions in dark grey.

Using this configuration the transmitting circuit is required to generate lower voltage pulses which would lead to a smaller and less power consuming design.

\section{Conclusions}

In this paper a full reconfigurable high-voltage transmitting circuit for CMUTs was designed and implemented in a $0.35 \mu \mathrm{m}$ high-voltage process. The pulsing frequency, driving speed, voltage levels and the transmitting, waiting and receiving time are easily adjustable externally making it suitable for CMUTs with very different specifications. The highest driving capabilities of the Tx circuit are a maximum voltage of $100 \mathrm{~V}$, a maximum pulse voltage swing of $50 \mathrm{~V}$ and a frequency of $5 \mathrm{MHz}$. Operating at these maximum specifications the transmitting circuit consumes $187.7 \mathrm{~mW}$ for a $15 \mathrm{pF}$ load. The area in the integrated circuit occupied by the Tx circuit is $0.938 \mathrm{~mm}^{2}$. In the future, several ideas and improvements to reduce the power consumption and area of the transmitting circuit are going to be tested and implemented.

\section{REFERENCES}

[1] Arif. S.Ergun, Goksen G. Yaralioglu and Butrus T. Khuri-Yakub, "Capacitive Micromachined Ultrasonic Transducers: Theory and Technology" in Journal of Aerospace Engineering, 2013, pp.74-87.

[2] G. Gurun, P. Hasler and F.L. Degertekin, "Front-End Receiver Electronics for High- Frequency Monolithic CMUT-on-CMOS Imaging Arrays" in IEEE Transactions on Ultrasonics, Ferroelectrics, and Frequency Control, 2011, Vol. 58, No. 8, pp.1658-1668.

[3] K. Chen, H-S. Lee, A.P. Chandrakasan and C.G. Sodini, "Ultrasonic Imaging Transceiver Design for CMUT: A Three-Level 30-Vpp PulseShaping Pulser With Improved Efficiency and a Noise-Optimized Receiver" in IEEE Journal of Solid-State Circuits, 2013, Vol. 48, No. 11, pp. 2734-2745.

[4] G. Gurun, P. Hasler and F.L. Degertekin, "A 1.5-mm Diameter SingleChip CMOS Front-End System with Transmit-Receive Capability for CMUTon- CMOS Forward-Looking IVUS" in IEEE International Ultrasonics Symposium Proceedings, 2011, pp.478-481.

[5] I.O. Wygant, X. Zhuang, D.T. Yeh, A. Nikoozadeh, . Oralkan, A.S. Ergun, M. Karaman and B.T. Khuri-Yakub, "An Endoscopic Imaging System Based on a Two-Dimensional CMUT Array: Real-Time Imaging Results" in IEEE Ultrasonic Symposium, 2005, pp.792-795. 